\title{
Significado de los valores profesionales en docentes y estudiantes de enfermería, UNMSM - 2014
}

Significance of professional values in nursing teachers and students, UNMSM - 2014

\author{
Martha Vera ${ }^{1, a}$, Rocío Cornejo ${ }^{1, b}$, Luisa Rivas ${ }^{1, c}$, Jessica Calizaya $^{2, d}$, \\ Oswaldo Zamora ${ }^{1, \mathrm{e}}$, Julissa Garcia ${ }^{1, \mathrm{e}}$ \\ Facultad de Medicina, Universidad Nacional Mayor de San Marcos, Lima, Perú. \\ ${ }^{2}$ Universidad Cesar Vallejo, Lima, Perú. \\ ${ }^{a}$ Profesor Principal, Doctor en Ciencias de la Salud, Miembro Permanente del Instituto de Ética en Salud, Departamento Académico de \\ Enfermeria; ${ }^{b}$ Profesor Principal, Doctor, Departamento Académico de Enfermería; ${ }^{\circ}$ Profesor Asociado, Magister, Departamento Académico \\ de Enfermería; ${ }^{d}$ Magister en Psicología Clínica y Salud, Profesor Facultad de Psicología; ${ }^{e}$ Internos de Enfermería, Escuela Académico \\ Profesional de Enfermeria, UNMSM
}

\begin{abstract}
Resumen
Objetivo. Interpretar el significado de los valores profesionales en docentes y estudiantes de la Escuela Académico Profesional de Enfermería de la Universidad Nacional Mayor de San Marcos. Metodología. Enfoque cualitativo, método descriptivo-interpretativo. E análisis fue ideográfico y nomotético. Muestra por criterio de saturación y entrevista a profundidad a 12 docentes, y 16 estudiantes de cuarto y quinto año de estudios constituidos en tres grupo focales. Resultados. Por objetivación y anclaje y dentro del entorno académico-administrativo, emergió la representación social del significado de los valores profesionales a través de cinco categorías y subcategorías. En Docentes: teniendo valores personales: amor, respeto, vocación profesional, responsabilidad y amistad, y valores inherentes al quehacer profesional: amor, responsabilidad, respeto, capacidad intelectual, justicia, y compromiso social. Estudiantes: teniendo valores personales: amor, respeto, amistad; y valores inherentes al quehacer profesional: desarrollando y consolidando la vocación; teniendo conocimiento científico, amor, respeto, amistad, responsabilidad, justicia, puntualidad. Conclusiones. Para docentes y estudiantes los valores profesionales significan tener como base valores personales de carácter moral y valores éticos inherentes al quehacer profesional, donde destacaron: amor, vocación, responsabilidad, respeto, amistad, justicia, conocimiento científico, aprendizaje de valores con ejemplo y modelo de docentes.
\end{abstract}

Palabras clave. Valores Profesionales Morales; Profesores; Estudiantes; Enfermería.

Abstract

Objective. To interpret the significance of professional values in students and teachers of the Nursing School at Universidad Nacional Mayor de San Marcos. Methods. Qualitative, descriptive-interpretative study. The analysis was ideographic and nomothetic. Sample by saturation criterion, and in depth interview to 12 teachers and 16 students of 4 th and 5th year of studies assembled in three focus groups. Results. By objectification and anchoring and within the academic and administrative environment, the social representation of the meaning of professional values emerged in five categories and subcategories. In Teachers: having personal values: love, respect, professional vocation, responsibility and friendship, and values inherent to the professional work: love, responsibility, respect, intellectual ability, justice, and social commitment. In Students: having personal values: love, respect, friendship; and values inherent to the professional work: developing and consolidating the vocation; taking scientific knowledge, love, respect, friendship, responsibility, justice, punctuality. Conclusions. For teachers and students professional values were based on personal moral and ethical values inherent to the professional work, including love, dedication, responsibility, respect, friendship, justice, scientific knowledge, learning values by example and teachers as models.

Keywords. Professionals-Moral Values; Teachers; Students; Nursing.

An Fac med. 2016;77(3):225-9/ http://dx.doi.org/10.15381/anales.v77i3.12404

\section{INTRODUCCIÓN}

Los valores morales son los pilares que sustentan los comportamientos y formas de vida de los seres humanos, pues "los valores en la sociedad actual son fruto de los cambios económicos y sociales que los transforman profundamente, porque no es una sociedad aislada, sino insertada en un mundo globalizado, de avances científicos y tecnológicos, aunque cada país tiene su escala de valores dependiendo de su cultura, desarrollo económico, religión" (1), "los valores son estándares validos de calidad de una persona o grupo social que surgen frecuentemente de necesidades y deseos" (2). Así mismo, "los valores morales son las necesidades más significativas de los hombres, traducidos en las tendencias del desarrollo social y expresadas en forma de ideal; son los intereses de la sociedad traducidos al plano de la conciencia moral" (3). "Son valores morales los que se dan en los actos humanos, solo si son realizados consciente y libremente por hombres a quienes se le puede atribuir responsabilidad moral, y para que se convierta en valor personal, debe ad- 
Esquema 2. Comparación entre los valores profesionales de docentes y estudiantes: convergencias en todo, excepto una divergencia.

\begin{tabular}{|c|c|c|c|}
\hline Categorías & Docentes & Estudiantes & Resultado \\
\hline $\begin{array}{l}\text { Valores personales relacionados } \\
\text { con la profesión }\end{array}$ & $\begin{array}{c}\text { Vocación, Amor, Respeto, Responsabilidad, } \\
\text { Amistad, Honestidad }\end{array}$ & $\begin{array}{l}\text { Amor, Vocación (consolidándose), Respeto, } \\
\text { Responsabilidad, Honestidad, Amistad }\end{array}$ & Convergencia al 100\% \\
\hline $\begin{array}{c}\text { Valores inherentes al quehacer } \\
\text { profesional }\end{array}$ & $\begin{array}{l}\text { Amor, Respeto a la dignidad de la persona, } \\
\text { Identidad profesional, Responsabilidad, } \\
\text { Solidaridad, Compromiso Social, } \\
\text { Conocimientos, Veracidad, Honestidad, } \\
\text { Justicia }\end{array}$ & $\begin{array}{c}\text { Amor en el cuidado, Respeto a la dignidad, } \\
\text { Identidad profesional, Responsabilidad, } \\
\text { Puntualidad, Solidaridad, Conocimientos, } \\
\text { Veracidad, Honestidad, Justicia }\end{array}$ & $\begin{array}{l}\text { Convergencia y única } \\
\text { Divergencia }\end{array}$ \\
\hline $\begin{array}{l}\text { Aprendizaje y enseñanza de los } \\
\text { valores profesionales }\end{array}$ & $\begin{array}{l}\text { Con el "ejemplo y siendo modelo" con } \\
\text { actuar ético como docente y enfermera } \\
\text { en la enseñanza del cuidado, en hospital, } \\
\text { centro de salud o el aula }\end{array}$ & $\begin{array}{l}\text { A través del docente (no solo de } \\
\text { enfermería) quien con su actuar ético da el } \\
\text { ejemplo y es un modelo a imitar para todos } \\
\text { y en todo }\end{array}$ & Convergencia en el $100 \%$ \\
\hline
\end{tabular}

Entorno o campo de la representación social: Es la misma EAPE y Facultad de Medicina en un ambiente físico, académico y social integrado por autoridades, personal administrativo, docentes y estudiantes; y todas las personas que tienen interrelación con los docentes y estudiantes de enfermería.

\section{DISCUSIÓN}

\section{ANÁLISIS E INTERPRETACIÓN (ANCLAJE)}

Como se observa en el esquema 1 (categorías 1 y 2 ), tanto docentes como estudiantes consideran que la base moral de los valores profesionales de enfermería son los valores personales, que "son las características fundamentales de una cultura, la cual se expresa a través del sistema de valores, y a través de la cual cobra sentido la vida en sociedad, al sistema eje de la vida social; es lo deseable en la vida del grupo ${ }^{(9)}$; Asimismo "cultura comprende todas las formas de comportamiento social creadas o adquiridas, que incluyen los modelos pautados de pensar, de sentir, de actuar y de creer de los grupos humanos" ${ }^{(9)}$. Por lo que los valores personales son parte constitutiva de la cultura personal de docentes y estudiantes con los cuales el grupo espera que sus miembros conformen sus actitudes y formas de actuar; ambos grupos coinciden al decir que la principal fuente de donde 'nacen' los valores personales es el hogar, la familia, comunidad, la sociedad. "Los valores personales son contemplados como principios que guían el comportamiento en respuesta a necesidades universales, y generalmente se manifiestan en la vida de la persona" ${ }^{(10)}$, y consideran como valores personales, al amor hacia el otro y así mismo, respeto, vocación, responsabilidad, entre otros. Valores que son morales, subjetivos y universales. El amor (está presente también en los valores inherentes al quehacer profesional) y amarse es "amar a otros y ser capaz de trascender; es lo opuesto a la indiferencia, pues el que ama quiere lo que es bueno para el objeto amado y se encuentra activamente preocupado y comprometido, se expresa por hechos y no por palabras y planteamientos y es un acto de voluntad, no de emociones" (11). El respeto, es otro valor que va unido al amor pues el que ama respeta al otro, que en este caso es el estudiante de enfermería, el usuario del cuidado y viceversa, pues "todas las personas necesitamos de los demás para desarrollarnos plenamente, el desarrollo personal depende en gran parte de las relaciones que cada uno establezca con sus semejantes y no tanto de los bienes materiales que se acumule. Por ello, los verdaderos bienes son aquellos que mejoren la convivencia" ${ }^{(12)}$. Otro valor es la vocación, que es amor por la profesión; en las docentes está plenamente desarrollada y en los estudiantes sienten que su vocación en unos está consolidada y en otros va en camino; esta se afianza a partir de la interrelación con sus pares, docentes y usuarios del cuidado que mientras más contacto tienen con el usuario sienten que eligieron bien, que aman lo que hacen, "la vocación es un trabajo en el sentido de una ocupación o de una tarea concreta en la que un individuo provee a su semejante lo mejor de sí. La vocación se entiende según el concepto de creación: somos creados para cuidar unos de otros mediante el trabajo" ${ }^{(13)}$. La identidad profesional es otro valor (está presente en los valores personales y los inherentes a la profesión), se construye a través del respeto y cumplimiento de normas, valores morales-éticos, de la Escuela Académico profesional de enfermería que la direccionan, y según el código de ética del Colegio de Enfermeros del Perú (CEP), por la identidad profesional la enfermera / docente "se encuentra identificada con los más altos valores morales y sociales de la humanidad y comprometida con aquellos que en lo particular propician una convivencia armónica de dignidad, justicia e igualdad enmarcados dentro del debe ser para una vida civilizada, para bien de la sociedad, de la profesión y usuarios de los servicios de enfermería" ${ }^{(14)}$.

La responsabilidad, valor con el que coinciden también, significa "asumir las consecuencias de nuestros actos. Ser 
responsable no es cumplir con algo impuesto por otros; la responsabilidad está relacionada con nuestra disposición para contribuir en una tarea común, es decir, poner en sintonía el interés propio con el interés colectivo" ${ }^{(12)}$. La amistad es otro valor, y sostienen que las personas al compartir ámbitos de actividades comunes, que en este caso es la Facultad de Medicina (EAPE), exige de ambos la necesidad de tomarse en cuenta, es decir, es imposible asumir una actitud indiferente hacia el otro; la Facultad debe ser una comunidad donde exista la verdadera amistad, entre docentes y estudiantes, Sostienen que no solo la amistad basta para entablar compañerismo. Es necesario un diálogo abierto, comunicación precisa y oportuna que permita el acercamiento y se puedan compartir los conocimientos, sentimientos, actividades, intereses, metas y objetivos comunes.

En la categoría 3 y 4 (esquema 1), según ambos grupos están los valores inherentes al quehacer profesional o valores éticos y son el amor hacia el otro, a la profesión; respeto a la dignidad humana, identidad profesional, solidaridad, responsabilidad, tenencia de conocimiento científico; honestidad $y$ justicia, valores que se fundamentan en la ética profesional, definida esta como "la actividad personal, puesta de manera estable y honrada al servicio de los demás y en beneficio propio, a impulso de la propia vocación y con la dignidad que le corresponde a la persona humana" ${ }^{(15)}$. La profesión "es una actividad social cooperativa cuya meta interna consiste en proporcionar a la sociedad un bien específico e indispensable para su supervivencia como sociedad humana, para lo cual se precisa el concurso de la comunidad profesional como tal" ${ }^{(16)}$. Valores profesionales de enfermería "son estándares que son promovidos por los códigos profesionales de conducta, los estándares de competencia profesional y la práctica de enfermería" (2). Los valores y la exigencia para su cumplimiento se plasma en el código de ética profesional, que en su primera disposición general dice “el Código de Ética y Deontología está constituido por un conjunto de principios, directivas y deberes que orientan y encausan el ejercicio profesional de la enfermera (o) peruana" ${ }^{(14)}$; y en la docencia e investigación, la enfermera debe "demostrar una sólida preparación científica, técnica y humanística, que asegure la formación profesional de alta calidad" (14), y garantizar un cuidado holístico e integral, puesto que al brindarlo la enfermera establece "una relación de persona a persona caracterizada por una relación afectiva, empática, de esperanza, comunicación y el uso terapéutico del otro yo" ${ }^{(13)}$, y considerar por ello como "factor del cuidado, a la formación de un sistema humanístico y altruista de valores de la enfermera, los que se aprenden en la vida pero que pueden recibir una gran influencia por parte de las enfermeras educadoras ${ }^{(13)}$.

En relación al valor del respeto a la dignidad de la persona: "para salvaguardar la dignidad de una persona, sea cual fuere su estado de salud, se debe brindar un cuidado espiritual ético, donde se demuestra apego, que es la obligación moral hacia los demás" (13), y según el Código de Ética del CEP, en su artículo primero y cuarto, obliga que la enfermera "brinde cuidados de enfermería respetando la dignidad humana y la singularidad de la persona, sin restricciones de índole social, de nivel económico, atributos personales, o naturaleza de los problemas de salud". Y "es deber de la enfermera (o) brindar atención eminentemente humana, oportuna, continua y segura, considerando la individualidad de la persona a quien cuida" ${ }^{(14)}$. En relación al valor profesional de la posesión del conocimiento científico que debe buscar y tener la enfermera, ambos grupos consideran que "la enfermería es una profesión que sustenta su actuar en un sistema de conocimientos reconocidos como ciencia ya constituida, la ciencia del cuidado" ${ }^{(13)}$, que tiene tradición e historia; "que en su recorrido ha desarrollado modelos, teorías de cuidado y discusiones filosóficas cuyo desarrollo se dio en un periodo que se caracteri- zó por la creación de modelos teóricos y conceptuales" (17). Recordar que actualmente la formación de la disciplina profesional, "constituye el ejercicio cotidiano de la paideia, que en palabras de los griegos era "la permanente construcción del saber en la vida, por la vida y para la vida desde unos principios éticos y filosóficos siempre en búsqueda de la verdad" ${ }^{(18)}$ y el enfermero debe ser "un docente de la nueva era, que no puede seguir conformándose con ser simplemente un mediador intermedio, meramente un ejecutor de un programa y transmisor de unos determinados conocimientos, sino que debe desarrollar competencias, tanto profesionales como personales, que lo conduzcan a sumergirse, de forma cada vez más profunda y activa en el proceso mismo de enseñanza-aprendizaje" ${ }^{(19)}$; y que "el docente debe promover su desarrollo profesional con actividades de formación permanente y de innovación e investigación educativa, para mantener un dominio permanente de los principios básicos de su materia o área, esforzándose por incorporar a su didáctica, los avances científicos, pedagógicos y didácticos oportunos" ${ }^{(20)}$. Justicia y solidaridad son valores profesionales considerados como muy importantes por ambos grupos, porque la justicia se pone en práctica al brindar el cuidado y tratar a todos por igual, sin diferencias por género, sexo, edad, raza, religión. La docente es justa y equitativa cuando enseña y evalúa al estudiante, solidariamente, respetando su dignidad como persona y su saber, y así el estudiante aprenderá a ser justo y solidario, con el ejemplo/modelo de sus docentes.

En el esquema 2, ambos grupos tienen una sola divergencia; las docentes consideran al valor del compromiso social como un valor importante, adquirido por su experiencia y conocimiento más profundo acerca de la realidad de la salud y necesidades de las personas, y los estudiantes consideran a la puntualidad, el valor fundamental para el cumplimiento del quehacer enfermero, refiriendo que la satisfacción de las necesidades del usuario no esperan. 
Tener en cuenta que la representación social no emerge sola de manera teórica a partir del discurso y su interpretación, sino se origina dentro de un entorno social, físico (esquema $1 \mathrm{y}$ 2), donde en el día a día interactúan sus miembros (docentes, estudiantes, autoridades y otros) para construir el conocimiento común sobre los valores, responsabilidades y funciones que le son propios, y es la Facultad y escuela de enfermería donde la docente es el modelo y ejemplo para los estudiantes, pues "la enfermera(o) debe actuar en coherencia con los principios éticos y deontológicos, constituyéndose en modelos de comportamiento en el desempeño humano y profesional" ${ }^{(13)}$.

En conclusión, para docentes y estudiantes los valores profesionales significan tener como base valores personales de carácter moral y valores éticos inherentes al quehacer profesional, donde destacan amor, identidad y vocación profesional, responsabilidad, veracidad, respeto, amistad, justicia, conocimiento científico, aprendizaje de valores con ejemplo y modelo de docentes no solo de enfermería sino de las otras disciplinas.

\section{REFERENCIAS BIBLIOGRÁFICAS}

1. Ramió A. Valores y actitudes profesionales. Estudio de la práctica profesional enfermera en Cataluña. Departamento de Sociología y Análisis de las
Organizaciones, Facultad de Ciencias Económicas y Empresariales. Universidad de Barcelona. España; 2005.

2. López A. Valores profesionales de enfermeros profesores según la percepción de los estudiantes de los últimos semestres de la carrera de enfermería en tres universidades de Bogotá. Tesis para obtener el Grado de Magister, Bogotá; 2002.

3. Amaro M. El método científico de enfermeria, e proceso de atención y sus implicaciones éticas y bioéticas. Revista Cubana de Enfermería. Habana. 2004. Disponible en: revistaenf@infomed.sld.cu.

4. Ortiz P. Desarrollo moral de la personalidad Facultad de Medicina, UNMSM, Lima, Perú. Separatas de clase teórica Doctorado en ciencias de la salud. 2002

5. Cárdenas L, Arana B, Monroy A, Garcia M. Cuidado profesional de enfermería. Federación Mexicana de Asociaciones de Facultades de Enfermeria, México, A.C. Jalisco. 2009;(85):91-2.

6. Dirección General de Profesiones. Secretaria de Educación Pública, Prototipo de Código de ética profesional. México DF; 1998.

7. Moscovici S. El fenómeno de las representaciones sociales en relaciones humanas, familia y sociedad. Buenos Aires, Argentina: Huemul S.A. 1981:p. 21

8. Vera M. Significado de la calidad de vida del adulto mayor para si mismo y para su familia. Tesis para optar el Grado de Doctor en ciencias de la salud. Lima, Perú. An Fac med. 2007;68(3):284-90.

9. Silva F. Antropologia: conceptos y nociones generales. Colección Biblioteca Universidad de Lima, Perú. Cuarta Edición; 1998.

10. Calvo A, Águila M, Salinas J. Valores laborales individualistas y colectivistas de enfermeras (o) en un entorno multicultural. ÍNDEX de enfermeria, Granada, España; octubre-noviembre 2008.

11. Rivera M. Teoria de la relación persona-persona de Joyce Travelbee, como sustento de la enseñanza de enfermeria. Escuela de Enfermeria, Universidad Pontificia Católica de Chile, 2003;14(1):99.

12. Arquetipo Grupo Editorial, Valores y actitudes. Primera Edición, Montevideo - Uruguay; 2004.

13. Marriner A, Raile M. Modelos y teorías de la enfermería. Barcelona, España: Elsevier Mosby, S.L.; 2011.

14. Colegio de Enfermeros del Perú, Código de Ética y Deontologia, DC 22315, 17 Octubre de 1978.
15. Ortiz C. La ética profesional en el ámbito de la profesión docente. Monografías.com S.A. publicado: lunes 6 de enero 2014.

16. Cortina A, Conill J. Presentación: El sentido de las profesiones. Navarra, España Edit. Verbo Divino; 2000:p.17

17. Pinto N, El cuidado como objeto del conocimiento de enfermeria. Facultad de Enfermeria, Universidad Nacional de Colombia, Revista Avances de Enfermeria, 2002;20(1). Disponible en: digital@ unal.edu.co.

18. Henao A, Núñez M, Quimbayo J. Ser profesor universitario en los programas del área de salud, Revista Aquichan. Abr 2010;10(1). CHIA, Colombia. Disponible en: Aquichan@Unisabana.edu.co.

19. Villanueva J. La filosofía y la formación docente hacia la construcción y consolidación de una praxis educativa más consciente, critica y participativa. Universidad Pedagógica Experimental Libertador, Caracas, Venezuela. LAURUS. Revista de Educación, Vol. (12). Disponible en: redalyc@redalyc.org.

20. Fonseca J. El desarrollo axiológico del profesorado y la mejora institucional. Universidad de ROVIRA I VIRGILI. Facultad de Ciencias de la Educación y Psicologia Departamento de Pedagogia. Programa Doctorado: Innovación y Sistema Educativo. Tarragona. España; 2003.

Artículo recibido el 6 de enero de 2016 y aceptado para publicación el 3 de mayo de 2016.

Trabajo ganador de la sección Miscelánea, XIV Jornadas Sanfernandinas de la Facultad de Medicina, Universidad Nacional Mayor de San Marcos, setiembre de 2015.

Correspondencia:

Dra. Martha Nicolasa Vera Mendoza

Departamento Académico de Enfermería, Facultad de Medicina, UNMSM.

Av. Grau 755, Lima 1, Perú

Correo-e: marthaveram@hotmail.com 